This manuscript is a non-peer reviewed preprint submitted to EarthArXiv. Please note that, we will submit it to a peer-review journal. Subsequent versions of this manuscript may have slightly different content. If accepted, the final version of this manuscript will be available via the 'Peer reviewed Publication DOI' link. Please feel free to contact any of the authors, we welcome feedback. 


\title{
Quantification of non-linear multiphase flow in porous media
}

\author{
Yihuai Zhang,* Branko Bijeljic, Ying Gao, Qingyang Lin, and Martin J. Blunt \\ Department of Earth Science and Engineering, Imperial College London, London SW7 2BP, United Kingdom
}

(Dated: August 20, 2020)

\begin{abstract}
We measure the pressure difference during two-phase flow across a sandstone sample for a range of injection rates and fractional flows of water, the wetting phase, during an imbibition experiment. We quantify the onset of a transition from a linear relationship between flow rate and pressure gradient to a non-linear power-law dependence. We show that the transition from linear (Darcy) to nonlinear flow and the exponent in the power-law is a function of fractional flow. We use energy balance to accurately predict the onset of intermittency for a range of fractional flows, fluid viscosities and three rock types, reconciling several literature datasets.
\end{abstract}

Introduction. - The flow of multiple fluids in porous materials occurs in a wide variety of important natural and engineered settings relevant for the understanding of geological $\mathrm{CO}_{2}$ storage, geothermal energy extraction, magma flow, oil and gas recovery, contaminant transport, flow in fuel cells, micro-fluidics in drug delivery, and the effectiveness of respirators and surgical masks, for instance [1-6]. It is assumed that the flow rate is proportional to the pressure gradient, governed by a Darcytype law $[1,7,8]$,

$$
q_{p}=-\frac{k_{r p} K}{\mu_{p}}\left(\nabla P_{p}-\rho_{p} g\right),
$$

where $q_{p}$ is the volume of phase $p$ flowing per unit area per unit time, $k_{r p}$ is the relative permeability, $K$ is the absolute permeability, $\mu_{p}$ is the viscosity, $\nabla P_{p}$ is the pressure gradient and $\rho_{p} g$ is the contribution of gravity.

In this work we will consider two-phase horizontal flow of a non-wetting phase, $n w$, and a wetting phase, $w$, where the gravitational term is zero. Also we will study steady-state flow where the capillary pressure (the pressure difference between the phases) does not vary across the sample: hence $\nabla P_{p}=\nabla P$ for both phases. In this case we can write the total Darcy flux $q_{t}=q_{n w}+q_{w}$ as:

$$
q_{t}=-\lambda_{t} K \nabla P
$$

where $\lambda_{t}$ is the total mobility: $\lambda_{t}=k_{r w} / \mu_{w}+k_{r n w} / \mu_{n w}$.

The relative magnitude of viscous and capillary forces can be encapsulated in the capillary number, defined for each phase as $C a_{p}=\mu_{p} q_{p} / \sigma$, where $\sigma$ is the interfacial tension between the phases. We also define a total capillary number following [9]:

$$
C a=\frac{q_{t}}{\sigma\left(\frac{1-f_{w}}{\mu_{n w}}+\frac{f_{w}}{\mu_{w}}\right)},
$$

where $f_{w}=q_{w} / q_{t}$ is the fractional flow. For a displacement with fixed fractional flow, but where the flow rate varies, Eq. (2) implies a linear relationship: $\nabla P \sim C a$.

It is well known that this linear law breaks down when, at the pore scale, viscous forces become comparable to

\footnotetext{
*Yihuai.Zhang@imperial.ac.uk
}

capillary forces with a threshold value of $\mathrm{Ca}$ of around $10^{-3}[1,10]$ : in this regime, the two phases flow together through the pore space with a fractional flow which is proportional to saturation. Recently, however, a body of research has demonstrated that multiphase flow in porous media has a complex dynamics even at low $\mathrm{Ca}$ where displacement is still dominated by capillary forces at the pore scale [11-16]. It has been proposed that there is a transition from capillary-dominated to an intermittent flow regime with a power-law dependence between pressure gradient and flow rate:

$$
\nabla P \sim C a^{a},
$$

with $1>a>0$ for $C a>C a^{i}$. Tallakstad et al. [17] conducted steady-state two-phase simultaneous flow experiments in a quasi-two-dimensional porous medium and found $a \approx 0.5$ in Eq. (4). Rassi et al. [18] measured an exponent $a$ between 0.3 and 0.45 depending on the fluid saturation from steady-state two phase flow in bead packs. Sinha et al. [19] also conducted experiments and simulations and proposed a power-law flow regime with an exponent $a=0.5$.

High-resolution X-ray tomography has allowed the fluid configurations to be imaged inside porous materials to interpret the physical origin of this non-linear regime, which is due to the intermittent occupancy of regions of the pore space by the two phases that facilitates flow $[9,20-22]$.

From a theoretical perspective, Sinha and Hansen [23] suggested that the exponent $a$ was around 0.5 and confirmed this behaviour using a dynamic pore-scale model. Roy et al. [24] proposed a size dependence of the nonlinear regime, such that for larger systems the onset of intermittency occurred at lower $\mathrm{Ca}$. In the limit of an infinite system this suggests that there is no linear Darcy regime at all. Gao et al. [21] quantified the threshold capillary number for the onset of intermittency $\mathrm{Ca}^{i}$ as approximately $10^{-5}$ and found $a=0.6$ from experiments of steady-state flow on water-wet Bentheimer sandstone for $f_{w}=0.5$; however, there were only eight data points. Overall, despite this body of theoretical, numerical and experimental work, there is no accurate quantification of when the transition to non-linear flow occurs and the relationship between pressure gradient and flow rate in this intermittent regime. 
In this paper, we study steady-state immiscible twophase flow through a water-wet Bentheimer sandstone sample with different flow rates (capillary number varies from $\sim 10^{-7}$ to $\sim 10^{-4}$ ) and fractional flows during an imbibition displacement $\left(f_{w}=0.2,0.4,0.5,0.6,0.8\right.$, and $1)$. We quantify the threshold capillary number for the onset of the power-law non-Darcy regime, Eq. (4) and measure the exponent $a$, which we find to be a function of fractional flow. We use energy balance to predict accurately the onset of non-Darcy flow as a function of capillary number and fractional flow, and for different rocks and viscosity ratios.

Steady-state flow experiments. - We performed experiments on a water-wet Bentheimer sandstone sample (5.97 $\mathrm{mm}$ diameter and $27.88 \mathrm{~mm}$ length) mounted in a specially designed core flooding system: details of the experimental apparatus can be found in the literature [20,21]. The wetting phase was $15 \mathrm{wt} \% \mathrm{KI}$ (potassium iodide) brine, and the non-wetting phase was $n$-decane, both injected by high precision ISCO pumps. To ensure uniform flow at the inlet, we used a dual injection port [25]; this provides a more even flow than T-junction injection used in previous studies which may have over-stated the degree of intermittent flow as a consequence [21].

During the flow, the pressure gradient between inlet and outlet of the sample was measured by a highprecision pressure transducer Keller PD-33X. The viscosity for the brine $\mu_{w}=0.821 \mathrm{mPas}$, the $n$-decane viscosity $\mu_{n w}=0.838 \mathrm{mPas}$ (PubChem, open chemistry database), while the interfacial tension was measured to be $\sigma=47$ $\mathrm{mN} / \mathrm{m}$. The absolute permeability for the sample was $K$ $=1.85( \pm 0.02) \times 10^{-12} \mathrm{~m}^{2}$, measured from the relationship between flow rate and pressure gradient when the core was fully saturated with brine. We then injected $n$ decane at $3 \mathrm{ml} / \mathrm{min}$ for $30 \mathrm{mins}$ to reach the initial brine saturation.

We started the two-phase flow experiment by injecting the two phases at a fractional flow $f_{w}$ of 0.2 at 0.04 $\mathrm{ml} / \mathrm{min}$ total flow rate $\left(\mathrm{Ca}=4.2 \times 10^{-7}\right)$; the pressure gradient was recorded after 10 hours when it stabilized, and then we gradually increased the flow rate from low to high and recorded the pressure gradients at steady-state. The time for the pressure gradient to become constant depended on the flow rates: it was up to ten hours for the low flow rates (less than $0.04 \mathrm{ml} / \mathrm{min}$ ) but as little as 5 minutes for high flow rate flooding (greater than 3 $\mathrm{ml} / \mathrm{min})$. The highest flow rate was $4.25 \mathrm{ml} / \mathrm{min}(C a=$ $\left.4.5 \times 10^{-5}\right)$.

Once we had reached the highest flow rate for a given fractional flow, we injected $n$-decane at $3 \mathrm{ml} / \mathrm{min}$ for 30 mins again to return to the initial saturation. We repeated the injection sequence at a series of increasing flow rates for other fractional flows: $0.4,0.5,0.6,0.7$, 0.8 , and 1 ; in total 178 flow experiments were conducted in this research.

Results.-The results, Fig. 1, show that $C a$ is proportional to the pressure gradient $\nabla P$ at low flow rates: $\nabla P \sim C a$ with $a=1$ is valid for all the fractional flows

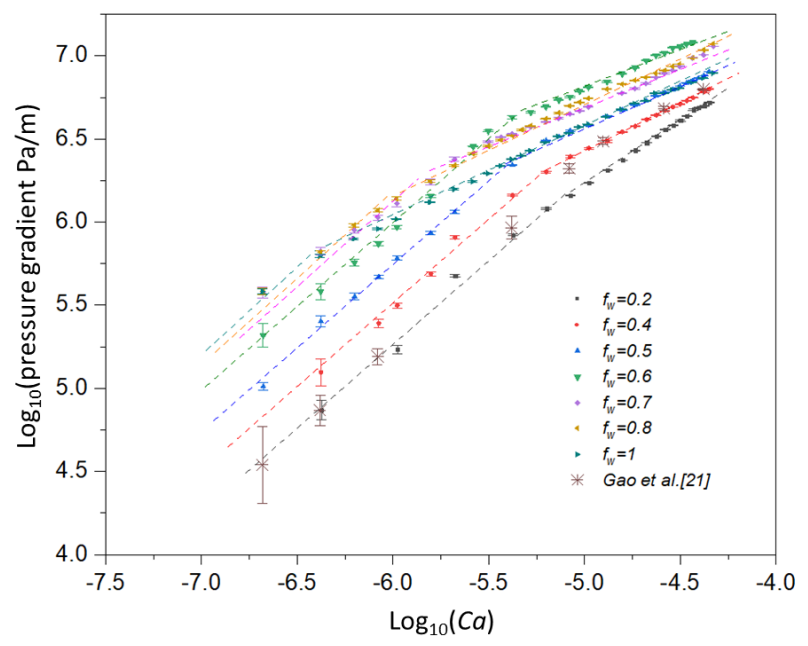

FIG. 1. The measured pressure gradients $\nabla P$ as a function of capillary number $C a$, Eq. (3), for different fractional flows, $f_{w}: 0.2,0.4,0.5,0.6,0.7,0.8$ and 1 ; also shown are the experimental results of Gao et al. [21], where $f_{w}=0.5$. The error bars reflect the standard deviation in the measurement of pressure gradient.

studied for sufficiently small $C a$. However, in all cases this is followed by a non-linear regime, Eq. (4). The exponent $a$ is a function of the fractional flow, see Table 1: the highest value $a=0.74 \pm 0.02$ occurs when $f_{w}=$ 0.2 , which displayed the lowest degree of intermittency defined as the deviation from a linear Darcy law; $f_{w}=$ 0.6 had the lowest exponent $a=0.44 \pm 0.02$ indicating a strong deviation from linear flow.

These observations are consistent with previous experiments where the rock and fluids were imaged at micron resolution, which concluded that intermittency, or a fluctuating pore occupancy, only constituted a small percentage of the total pore volume for $f_{w}<0.5$ [22]. Moreover, in our experiments, when $f_{w}<0.5$, the pressure gradient increased with fractional flow indicating that the total mobility decreases; for $f_{w}>0.5$, a more complex behaviour emerged, where curves of $\nabla P$ as a function of $C a$ for constant $f_{w}$ crossed each other, indicating a significant increase in mobility with flow rate for the intermediate fractional flows, were both phases compete to occupy high-conductivity paths through the pore space [21].

The threshold capillary number $C a^{i}$ between the linear Darcy regime and intermittent flow, Table 1, is also a function of the fractional flow: $C a^{i}$ decreased from $\sim$ $10^{-5.1}$ to $\sim 10^{-6.4}$ as the fractional flow $f_{w}$ increased from 0.2 to 1 . Thus, higher fractional flows have a lower critical flow rate $q_{t}^{i}$ for the onset of intermittency. Note that the results for a fractional flow of 1 represent the capillary desaturation process [10]; at steady-state, there is only one mobile phase.

Quantification of the transition from linear to intermittent flow.-We assume that we see the onset of intermittency when the energy, associated with the in- 
TABLE I. Summary of the exponent $a$ for $\nabla P \sim C a^{a}$, threshold capillary number $C a^{i}$, the associated non-wetting phase capillary number $C a_{n w}^{i}$, and the wetting phase capillary number $C a_{w}^{i}$ for the onset of intermittency, see Fig. 1.

\begin{tabular}{cccccc}
\hline \hline Fractional flow $\left(f_{w}\right)$ & Exponent $a\left(C a<C a^{i}\right)$ & $C a^{i}$ & $C a_{n w}^{i}$ & $C a_{w}^{i}$ & Exponent $a\left(C a>C a^{i}\right)$ \\
\hline 0.2 & 1 & $\sim 10^{-5.1}$ & $\sim 10^{-5.2}$ & $\sim 10^{-5.8}$ & $0.74 \pm 0.02$ \\
0.4 & 1 & $\sim 10^{-5.2}$ & $\sim 10^{-5.4}$ & $\sim 10^{-5.6}$ & $0.57 \pm 0.02$ \\
0.5 & 1 & $\sim 10^{-5.3}$ & $\sim 10^{-5.5}$ & $\sim 10^{-5.5}$ & $0.48 \pm 0.02$ \\
0.6 & 1 & $\sim 10^{-5.4}$ & $\sim 10^{-5.8}$ & $\sim 10^{-5.6}$ & $0.44 \pm 0.02$ \\
0.7 & 1 & $\sim 10^{-5.5}$ & $\sim 10^{-6.0}$ & $\sim 10^{-5.7}$ & $0.47 \pm 0.02$ \\
0.8 & 1 & $\sim 10^{-5.7}$ & $\sim 10^{-6.3}$ & $\sim 10^{-5.8}$ & $0.56 \pm 0.02$ \\
1 & 1 & $\sim 10^{-6.4}$ & - & $\sim 10^{-6.4}$ & $0.66 \pm 0.02$ \\
\hline \hline
\end{tabular}

TABLE II. Summary of the pore geometry properties, fluid viscosities and calculated values of $Y^{i}$, Eq. (8), for different porous media: the predicted onset of intermittency is compared to the experimental results in Fig. 3

\begin{tabular}{ccccccc}
\hline \hline Porous medium & $\phi$ & $r(\mu m)$ & $K\left(10^{-12} m^{2}\right)$ & $l(\mu m)$ & $\mu_{n w}(\mathrm{mPas})$ & $\mu_{w}(\mathrm{mPas})$ \\
\hline Bentheimer [15] & 0.20 & 24 & 1.85 & 150 & 0.021 & 0.642 \\
Bentheimer [20] & 0.20 & 24 & 1.85 & 150 & 0.838 & 0.821 \\
Estaillades [9, 25] & 0.295 & 7.5 & 0.15 & 63 & 0.838 & $10^{-6.5}$ \\
Estaillades [9] & 0.295 & 7.5 & 0.15 & 63 & 0.021 & 0.821 \\
Estaillades [9] & 0.295 & 7.5 & 0.15 & 63 & 3.45 & 0.821 \\
Glass beads [13] & 0.41 & 12.5 & 1.43 & 25 & 16.8 & $10^{-5.7}$ \\
Glass beads [13] & 0.41 & 12.5 & 1.43 & 25 & 16.8 & $10^{-7.3}$ \\
Glass beads [13] & 0.41 & 21 & 3.96 & 41 & 16.8 & $10^{-5.1}$ \\
\hline \hline
\end{tabular}

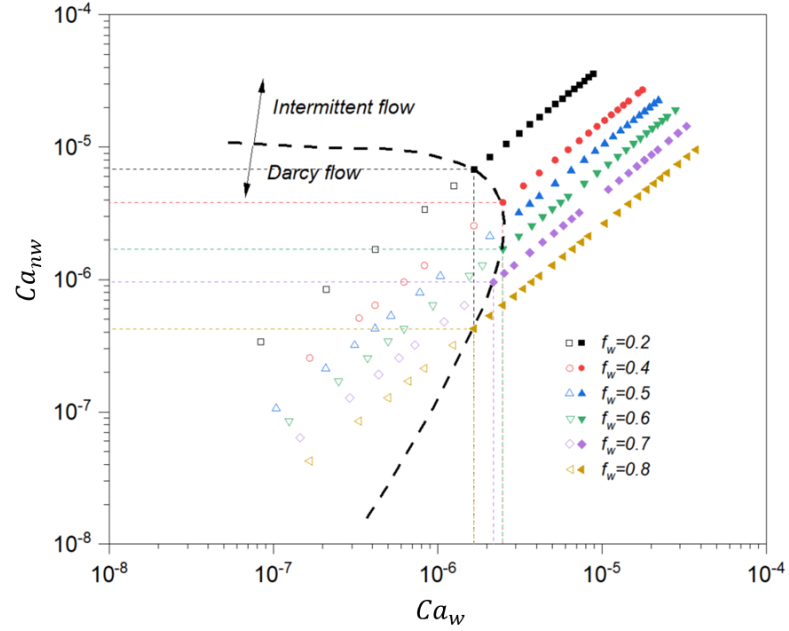

FIG. 2. The phase diagram of the transition from Darcy flow (empty symbols) to intermittent flow (filled symbols) as a function of non-wetting phase capillary number $C a_{n w}^{i}$ and wetting phase capillary number $C a_{w}^{i}$; the dashed line is the predicted threshold using Eqs. (6) and (7).

jection of fluids over a characteristic length $l$, related to the distance between pores, is first matched by the change in surface energy needed to create a fluid meniscus $[1,21,26]$. The interfacial energy required to form an interface between the fluids inside a single pore is of order $\sigma r^{2}$ where $r$ is a typical pore radius. The mechanical $(P d V)$ work occurs over a pore volume of $\phi l^{3}$ where $\phi$ is the porosity. The change in pressure across this length is $-l \nabla P$ where $\nabla P$ is the pressure gradient. Hence intermittency first occurs when $\sigma r^{2} \sim-\phi l^{4} \nabla P$. We now need to estimate $\nabla P$ over a typical pore length. We can assume a Darcy-like flow, although we need to note that we apply it at the pore scale. In imaging experiments, intermittency is caused by the non-wetting phase periodically finding more conductive pathways through the pore space $[9,21]$. We assume that since both phases have to move, the pressure gradient necessary to allow intermittency is controlled by the total threshold flow rate $q_{t}^{i}$. The limiting mobility is assumed to be $\left(1-f_{w}\right) / \mu_{w}$, governed by the flow of the wetting phase into and out of pores filled with non-wetting phase with an effective relative permeability, at least in the viscous-flow limit, of $1-f_{w}$, or the non-wetting phase fractional flow. Hence from Eq. (1) we estimate $\nabla P \approx-\mu_{w} q_{t}^{i} / K\left(1-f_{w}\right)$. We then expect the onset of intermittency when:

$$
\sigma r^{2}=\frac{\mu_{w} q_{t}^{i} \phi l^{4}}{K\left(1-f_{w}\right)}
$$

This can be rearranged to write the threshold capillary numbers $C a_{w}^{i}=\mu_{w} f_{w} q_{t}^{i} / \sigma$ and $C a_{n w}^{i}=\mu_{n w}\left(1-f_{w}\right) q_{t}^{i} / \sigma$ as follows:

$$
C a_{n w}^{i}=Y^{i}\left(1-f_{w}\right)^{2}
$$

and

$$
C a_{w}^{i}=Y^{i} f_{w}\left(1-f_{w}\right) \frac{\mu_{w}}{\mu_{n w}},
$$

where the dimensionless number $Y^{i}$ is defined by:

$$
Y^{i}=\frac{\mu_{n w}}{\mu_{w}} \frac{K r^{2}}{\phi l^{4}} .
$$




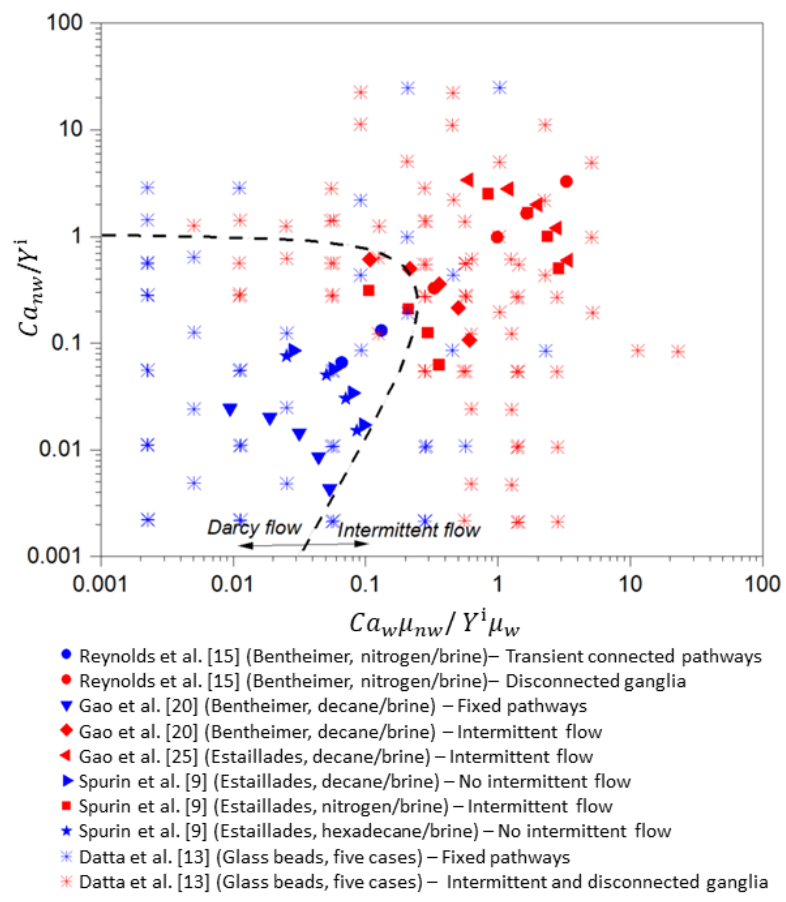

FIG. 3. The re-plotted phase diagram of the transition from connected pathway flow (Darcy flow, Eqs. (1) and (2)) to no connected pathways (intermittent flow, Eq. (4) using literature data $[9,13,15,20,25]$ where different viscosity fluids were injected into different rocks; full details can be found in Table 2 . In these experiments the pressure gradient was not measured; instead the onset of intermittent flow was estimated from the pore-scale dynamics as described in the legend. The dashed line is the predicted threshold from Eqs. (6) and (7).

In our experiemnts, for Bentheimer sandstone, the porosity $\phi$ is 0.2 , the mean pore radius $r$ is $24 \mu \mathrm{m}$ [1], while to capture the onset of intermittency $l$ has a value of approximately $150 \mu \mathrm{m}$ [21]: $l$ is the mean pore-to-pore distance obtained from a pore-network analysis of the pore structure [27]. Then using the fluid viscosities in this experiment we calculate $Y^{i} \approx 10^{-5}$.

In Fig. 2, we delineate the threshold between Darcylike and intermittent flow using Eqs. (6) and (7) which provides an accurate prediction compared to the values in Table 1. Note, however, that Eqs. (6) and (7) are only valid when we strictly have multiphase flow with $1>f_{w}>0$ and cannot be applied for $f_{w}=1$ or 0 .

Our experiments were performed for one set of fluids with almost equal viscosities. To test the applicability of our theoretical result for different fluids, we apply Eq. (8) to the data from Reynolds et al. [15]. As in this work the rock studied was Bentheimer sandstone, so that the geometric terms in Eq. (8) are the same. We show again that we make accurate predictions where we calculate $Y^{i}=10^{-6.5}$, for nitrogen and brine injection: the threshold line (Fig. 3) between Darcy and intermittent flow is consistent with the X-ray pore scale images described in [15].

Furthermore, we also apply our theory to a different rock sample, an Estaillades carbonate rock with both low (nitrogen and brine, $\mu_{n w} / \mu_{w}=0.026$ ) and high (hexadecane and brine, $\mu_{n w} / \mu_{w}=4.21$ ) viscosity ratio fluids; here $\phi=0.295, r=7.5 \mu \mathrm{m}, K=1.49 \times 10^{-13} \mathrm{~m}^{2}$ and $l$ has a value of approximately $63 \mu \mathrm{m}$ (here the pore length is assumed to be the inverse of the cube root of the number of pores per unit volume, while the radius is found from a pore network analysis) [1]: Eq. (8) is used to calculate $Y^{i}=10^{-7.3}$ and $10^{-5.1}$ respectively. The predicted thresholds are consistent with the results of Spurin et al. [9] where X-ray scanning results showed that the low viscosity ratio fluid system displayed strong intermittency, but no intermittency was observed at the pore scale for the high fluid viscosity ratio case although the total capillary numbers were similar.

We finally apply our theory to other data in the literature for Bentheimer sandstone, Estaillades limestone and glass bead packs $[13,20,25]$ : details are provided in Fig. 3 and Table 2. For a bead pack, we estimate $l=$ $D \phi /(1-\phi)[1,28,29]$, where $D$ is the bead diameter; the radius, $r$, is the half of the average pore size. The permeability $K$ can be estimated from $K=\phi^{3} D^{2} / 180(1-\phi)^{2}$ [1]. In all cases we predict the onset of intermittency, as demonstrated in Fig. 3 with only a few discrepancies for the bead pack data. Note, however, that in the experiments pressure gradient was not measured, and so the onset of intermittency was determined from an analysis of the pore-scale dynamics as described in the legend of the figure.

Conclusions.-We have measured the pressure gradient as a function of flow rate for different fractional flows of oil and brine through a small sample of Bentheimer sandstone. We have observed the Darcy flow regime and the transition to non-Darcy or intermittent flow. At low flow rates, the flow follows a standard linear Darcy law, while for higher flow rates we see $\nabla P \sim C a^{a}$. We proposed a relationship for the threshold capillary number for the onset of intermittent flow, Eqs. (6) and (7), which accurately matched the experimental results and is applicable to the different viscosity ratio fluids and different rock types, reconciling a large body of experimental results in the literature.

Future work could include the study of flow at different viscosity ratios, system lengths, mixed-wet and oil-wet media and different types of porous material.

\section{ACKNOWLEDGMENTS}

We gratefully acknowledge funding from the Shell Digital Rocks programme at Imperial College London. 
[1] M. J. Blunt, Multiphase flow in permeable media: A porescale perspective (Cambridge University Press, 2017).

[2] M. A. Gjennestad, M. Winkler, and A. Hansen, Pore network modeling of the effects of viscosity ratio and pressure gradient on steady-state incompressible two-phase flow in porous media, Transport in Porous Media , 1 (2020).

[3] T. Pak, I. B. Butler, S. Geiger, M. I. van Dijke, and K. S. Sorbie, Droplet fragmentation: 3d imaging of a previously unidentified pore-scale process during multiphase flow in porous media, Proceedings of the National Academy of Sciences 112, 1947 (2015).

[4] C. Reynolds and S. Krevor, Characterizing flow behavior for gas injection: Relative permeability of co2-brine and n2-water in heterogeneous rocks, Water Resources Research 51, 9464 (2015).

[5] Y. Zhang, M. Lebedev, Y. Jing, H. Yu, and S. Iglauer, In-situ x-ray micro-computed tomography imaging of the microstructural changes in water-bearing medium rank coal by supercritical co2 flooding, International Journal of Coal Geology 203, 28 (2019).

[6] B. Zhao, A. A. Pahlavan, L. Cueto-Felgueroso, and R. Juanes, Forced wetting transition and bubble pinch-off in a capillary tube, Physical review letters 120, 084501 (2018).

[7] M. Muskat and M. W. Meres, The flow of heterogeneous fluids through porous media, Physics 7, 346 (1936).

[8] M. Muskat, The flow of homogeneous fluids through porous media, Soil Science 46, 169 (1938).

[9] C. Spurin, T. Bultreys, B. Bijeljic, M. J. Blunt, and S. Krevor, Mechanisms controlling fluid breakup and reconnection during two-phase flow in porous media, Physical Review E 100, 043115 (2019).

[10] L. W. Lake, Enhanced oil recovery (Old Tappan, NJ; Prentice Hall Inc., 1989).

[11] R. T. Armstrong and S. Berg, Interfacial velocities and capillary pressure gradients during haines jumps, Physical Review E 88, 043010 (2013).

[12] S. Berg, H. Ott, S. A. Klapp, A. Schwing, R. Neiteler, N. Brussee, A. Makurat, L. Leu, F. Enzmann, J.-O. Schwarz, et al., Real-time 3d imaging of haines jumps in porous media flow, Proceedings of the National Academy of Sciences 110, 3755 (2013).

[13] S. S. Datta, J.-B. Dupin, and D. A. Weitz, Fluid breakup during simultaneous two-phase flow through a three-dimensional porous medium, Physics of Fluids 26, 062004 (2014).

[14] S. S. Datta, T. Ramakrishnan, and D. A. Weitz, Mobilization of a trapped non-wetting fluid from a threedimensional porous medium, Physics of Fluids 26, $022002(2014)$.

[15] C. A. Reynolds, H. Menke, M. Andrew, M. J. Blunt, and S. Krevor, Dynamic fluid connectivity during steadystate multiphase flow in a sandstone, Proceedings of the National Academy of Sciences 114, 8187 (2017).

[16] M. Rücker, S. Berg, R. Armstrong, A. Georgiadis, H. Ott,
A. Schwing, R. Neiteler, N. Brussee, A. Makurat, L. Leu, et al., From connected pathway flow to ganglion dynamics, Geophysical Research Letters 42, 3888 (2015).

[17] K. T. Tallakstad, H. A. Knudsen, T. Ramstad, G. Løvoll, K. J. Måløy, R. Toussaint, and E. G. Flekkøy, Steadystate two-phase flow in porous media: statistics and transport properties, Physical review letters 102, 074502 (2009).

[18] E. M. Rassi, S. L. Codd, and J. D. Seymour, Nuclear magnetic resonance characterization of the stationary dynamics of partially saturated media during steadystate infiltration flow, New Journal of Physics 13, 015007 (2011).

[19] S. Sinha, A. T. Bender, M. Danczyk, K. Keepseagle, C. A. Prather, J. M. Bray, L. W. Thrane, J. D. Seymour, S. L. Codd, and A. Hansen, Effective rheology of twophase flow in three-dimensional porous media: experiment and simulation, Transport in porous media 119, 77 (2017).

[20] Y. Gao, Q. Lin, B. Bijeljic, and M. J. Blunt, X-ray microtomography of intermittency in multiphase flow at steady state using a differential imaging method, Water resources research 53, 10274 (2017).

[21] Y. Gao, Q. Lin, B. Bijeljic, and M. J. Blunt, Pore-scale dynamics and the multiphase darcy law, Physical Review Fluids 5, 013801 (2020).

[22] C. Spurin, T. Bultreys, B. Bijeljic, M. J. Blunt, and S. Krevor, Intermittent fluid connectivity during twophase flow in a heterogeneous carbonate rock, Physical Review E 100, 043103 (2019).

[23] S. Sinha and A. Hansen, Effective rheology of immiscible two-phase flow in porous media, EPL (Europhysics Letters) 99, 44004 (2012).

[24] S. Roy, S. Sinha, and A. Hansen, Immiscible two-phase flow in porous media: Effective rheology in the continuum limit, arXiv preprint arXiv:1912.05248 (2019).

[25] Y. Gao, A. Q. Raeini, M. J. Blunt, and B. Bijeljic, Pore occupancy, relative permeability and flow intermittency measurements using x-ray micro-tomography in a complex carbonate, Advances in Water Resources 129, 56 (2019).

[26] L. Cueto-Felgueroso and R. Juanes, A discrete-domain description of multiphase flow in porous media: Rugged energy landscapes and the origin of hysteresis, Geophysical Research Letters 43, 1615 (2016).

[27] A. Q. Raeini, B. Bijeljic, and M. J. Blunt, Generalized network modeling: Network extraction as a coarse-scale discretization of the void space of porous media, Physical Review E 96, 013312 (2017).

[28] S. Whitaker, The method of volume averaging, Vol. 13 (Springer Science \& Business Media, 2013).

[29] G. M. Porta, B. Bijeljic, M. Blunt, and A. Guadagnini, Continuum-scale characterization of solute transport based on pore-scale velocity distributions, Geophysical Research Letters 42, 7537 (2015). 